\title{
AVALIAÇÃO DA ALTERABILIDADE DAS ROCHAS DA FACHADA DO MUSEU CASA DE RUI BARBOSA
}

\author{
Daniele Pereira da Silva Dalto ${ }^{1}$, Paula Ribeiro Dias Mascarenhas ${ }^{2}$, Kátia Leite Mansur ${ }^{3}$, Roberto \\ Carlos da Conceição Ribeiro ${ }^{4}$
}

1 - Engenheira Química, M.Sc., CETEM; 2 - Estudante de Geologia, UFRJ; 3 - Professora/Geóloga, D.Sc., UFRJ; 4 - Engenheiro Químico, D.Sc., CETEM ddalto@cetem.gov.br

Resumo: O Museu Casa de Rui Barbosa, cuja construção foi feita no ano de 1850, localiza-se no bairro de Botafogo na cidade do Rio de Janeiro e faz parte da Fundação Casa de Rui Barbosa tombado pelo IPHAN. O edifício que abriga o Museu foi residência de Ruy Barbosa de Oliveira que dentre outras atribuições foi um jurista, escritor e político brasileiro de grande importância para o país. Devido a importância de cunho histórico e cultural de sua residência, o objetivo do presente trabalho é a caracterização e a avaliação da alterabilidade das rochas das fachadas da Casa de Rui Barbosa por meio de ensaios não destrutivos in situ. Para tal, foram realizados ensaios de avaliação de dureza, análise química por meio da fluorescência de raios-X portátil (FRX) e colorimetria. Foi feito também o mapeamento litológico, identificação das morfologias de alteração e amostragem da água de lavagem de locais alterados para avaliação química dos sais e sujidades utilizando técnica de espectrometria ICP-OES e análise de pH. A maioria das rochas ornamentais das fachadas são, possivelmente, gnaisses leptiníticos, e alguns apresentam alterações como modificação cromática, perda de massa, fissuras e esfoliação, porém há ainda a presença de gnaisses facoidais. A análise de FRX corrobora com a hipótese das rochas serem leptinitos devido ao alto teor de silício (50-85\%) e alumínio (12-32\%), além de ferro (1-5\%), indicando presença de granada almandina. Foi possível observar que há uma leve alteração cromática nos corpos avaliados. A análise química das águas de lavagem apresentou teores consideráveis de íons sódio e cloro, ambos com cerca de $7 \mathrm{mg}$.L-1, que podem estar relacionados com a proximidade do Museu à Baía de Guanabara, e enxofre com valores de S-2 e SO4-2, superior a 70 mg.L-1 e 140 mg.L-1, respectivamente, podendo estar relacionado à poluição oriunda do intenso tráfego de veículos na rua em que se encontra a casa. Os resultados apontam que existe um processo de degradação contínuo destas rochas, sendo influenciado por fatores climáticos e, também, pela sua localização.

Palavras Chave: rochas ornamentais, alterabilidade, Museu Casa de Rui Barbosa

Abstract: ALTERABILITY EVALUATION OF THE ROCK FACADE FROM CASA DE RUI BARBOSA MUSEUM. Casa Rui Barbosa Museum, was built in 1850, located in Botafogo in the city of Rio de Janeiro, and part of Foundation Casa de Rui Barbosa - IPHAN. The building was the residence of Ruy Barbosa de Oliveira who among other duties was a lawyer, a writer and a politician of great importance to Brazil. Because of the importance of historical and cultural nature, the aim of this study is the evaluation of the alterability of the stones of the facades of the Casa Rui Barbosa Museum through non-destructive testing in situ, hardness evaluation, chemical analysis by fluorescence-ray portable $X(F R X)$ and colorimetric analysis. Also the lithological mapping, identification of alterability and chemical evaluation of the salts and dirtiness of the wash water using ICP-OES spectrometry, moreover $\mathrm{pH}$ analysis. Most ornamental stones of the facades are possibly leptynite, and some feature changes such as color change, weight loss, cracks and exfoliation, but there is still the presence of augen gneisses. The XRF analysis supports the hypothesis that stones are leptynite due to the high silicon content (50-85\%) and aluminum (12$32 \%)$, and iron (1-5\%), indicating the presence of almadine garnet. A slight color change was observed in the stones. Chemical analysis of the water showed significant levels of sodium and chloride ions, both with approximately $7 \mathrm{mg}$.L-1, which may be related to the proximity of the Guanabara Bay, and high values of S-2and SO4-2, above $70 \mathrm{mg} . \mathrm{L}-1$ and $140 \mathrm{mg} . \mathrm{L}-1$, respectively, and may be related to pollution from the heavy vehicle traffic on the street where the Museum is located. The results showed that there is a continuous degradation process of these stones, influenced by weathering and the location.

Keywords: dimension stones, weathering, Casa de Rui Museum

\section{INTRODUÇÃO}

O Museu Casa de Rui Barbosa, localizado no bairro de Botafogo, na cidade do Rio de Janeiro, integra a Fundação Casa de Rui Barbosa, uma instituição vinculada aos Ministérios da Educação e da Cultura e que tem como missão promover a preservação e a pesquisa da memória e da produção literária e humanística, bem como congregar iniciativas de reflexão e debate acerca da cultura brasileira. A construção data de 1850 e foi residência do jurista, escritor e político brasileiro Ruy Barbosa de Oliveira, que dentre outras contribuições ao país, foi coautor da Constituição da Primeira República e Presidente da Academia Brasileira de Letras, após a morte de Machado de Assis. O valor desta construção não se delimita apenas por ter abrigado Ruy Barbosa, mas também por ter sido cenário de grandes eventos sociais e políticos da época. A

doi: $10.18285 /$ geonomos.v24i2.845 residência é constituída por paredes externas com função estrutural, compostas por alvenarias mistas de tijolos e pedras, paredes divisórias internas em tabiques estucados, estrutura de pisos, forros e telhado em madeira, e cobertura em telhas cerâmicas do tipo francesa (NPArq, 2015).

\section{OBJETIVOS}

O presente trabalho tem como objetivo a caracterização e aavaliação da degradação das rochas da fachada principal sul da Casa de Rui Barbosa, localizada na cidade do Rio de Janeiro, por meio de ensaios não destrutivos in situ.

\section{METODOLOGIA}

Na Figura 1 está disposta a localização em planta baixa das fachadas do Museu. A fachada sul, na Figura 2, está voltada para a Rua São Clemente, uma das ruas mais movimentadas e urbanizadas do bairro de Botafogo, com alto tráfego de pedestres e 
automóveis. Na Figura 2(i) está em destaque as janelas de estudo do presente trabalho (SJ3 e SJ4), e na Figura 2(ii) está a divisão e subdivisão em corpos de rocha de cada janela e porta para um melhor estudo.

\subsection{Mapeamento litológico e identificação das} morfologias de alteração

Foi realizado o mapeamento litológico e identificação das morfologias de alteração in situ das rochas ornamentais (portas e janelas) que compõem a fachada principal sul.

\subsection{Avaliação de dureza}

A dureza foi medida in situ pelo método dinâmico de rebote (rebound), com o equipamento Equotip 550 Leeb da marca Proceq, e é padronizado de acordo com o ASTM A 956.

\subsection{Análise química pela fluorescência de raios-} X (FRX)

A análise química das rochas foi realizada por meio de ensaios não destrutivos in situ com o equipamento de fluorescência portátil S1 Turbo SD da marca Bruker.

\subsection{Análise colorimétrica e de brilho}

A análise colorimétrica e de brilho das rochas foi realizada utilizando um colorímetro Guide Sphere Gloss da marca BYK obtendo valores correspondentes às cores nos eixos $a, b$ e $L$, além do brilho (G).

\subsection{Análise de sais e sujidades}

A coleta da água de lavagem foi realizada com auxílio de uma escova de cerdas macias, potes coletores de polipropileno estéreis e água deionizada. Foram amostrados pontos em que se podia notar alterações na superfície da rocha. Os principais elementos constituintes das amostras coletadas foram determinados por espectrometria de emissão ótica com plasma induzido (ICP-OES).

\subsection{Análise de pH das águas de lavagem}

A análise de $\mathrm{pH}$ foi realizada utilizando o pHmetro digital da marca ThermoScientific, modelo Orion 4-Star.

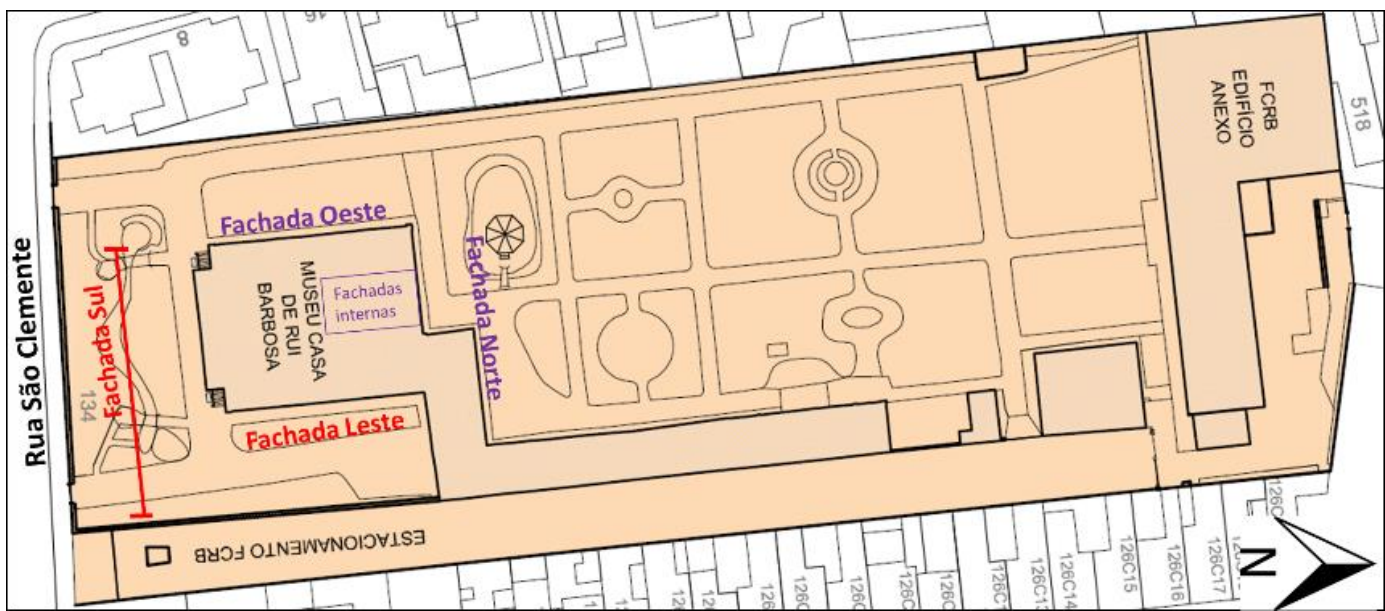

Figura 1. Vista aérea do Museu Casa de Rui Barbosa (NPArq, 2015).

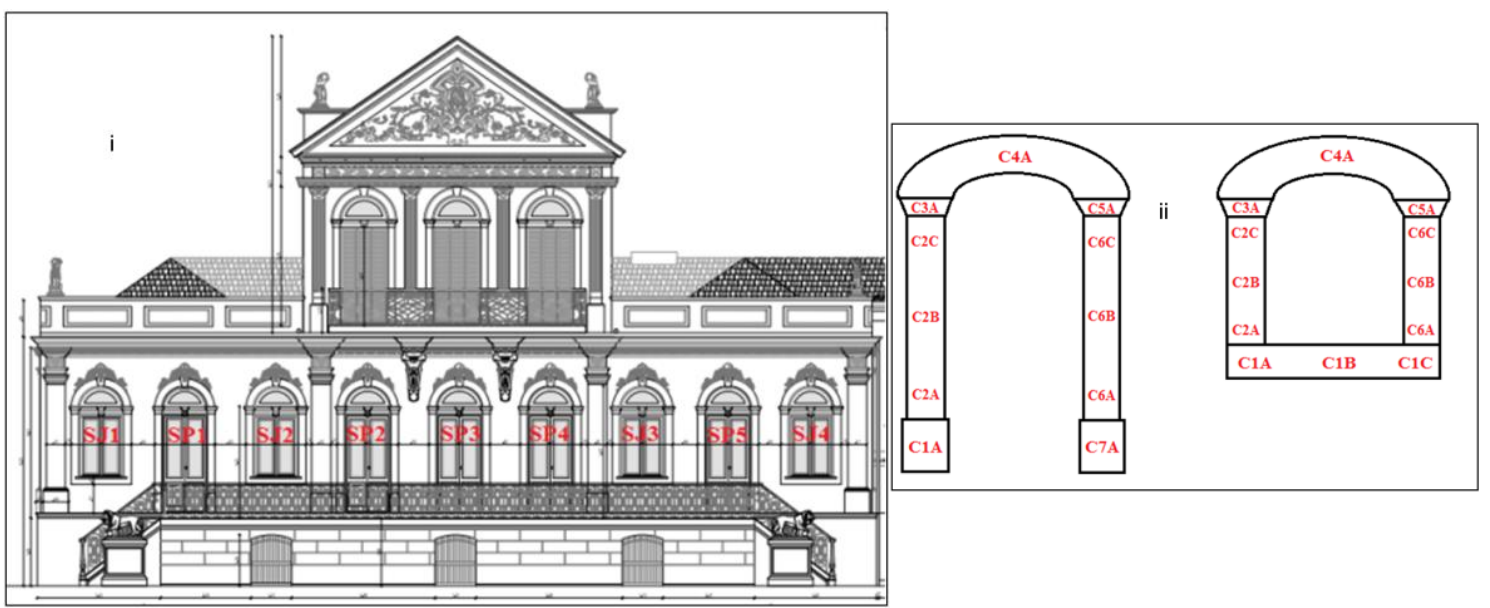

Figura 2. (i) planta da fachada principal com a nomenclatura de suas portas e janelas (ii) nomenclatura dos corpos de rocha que as compõem e suas subdivisões. 


\section{RESULTADOS E DISCUSSÃO}

\subsection{Mapeamento litológico e identificação das morfologias de alteração}

Foi possível observar que a maioria das rochas ornamentais do Museu Casa Rui Barbosa que compõem as portas e janelas da Fachada Sul são, possivelmente, gnaisses leptiníticos (ou leptinitos), enquanto nas fachadas restantes predomina 0 gnaisse facoidal (augen gnaisse), e alguns apresentam modificação cromática, perda de massa, fissuras e esfoliação. Normalmente a alteração cromática está relacionada a fatores climáticos, como a exposição à luz ultravioleta oriunda do sol, à água e à umidade. Já a perda de massa, fissuras e esfoliação podem estar relacionadas além do clima, com a localização, pois o Museu se localiza em uma rua com tráfego intenso de pessoas e veículos.

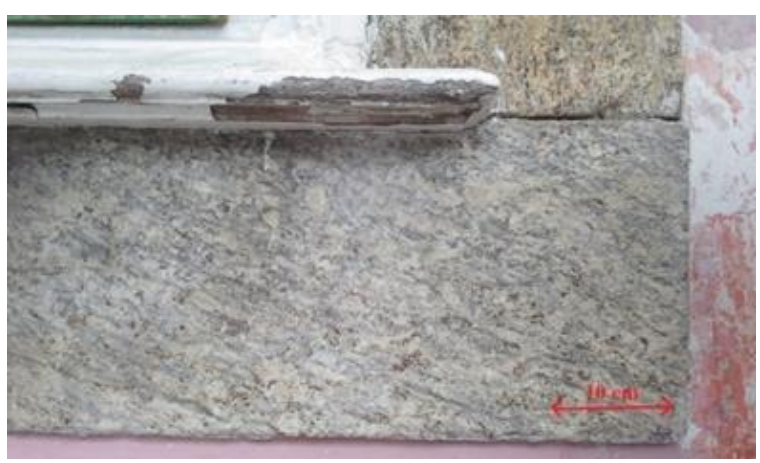

Figura 3. Rocha da Janela SP4 da fachada sul.

\subsection{Avaliação de dureza}

A fachada principal sul apresentou uma variação significante nos valores de dureza, conforme Tabela 1 e 2, mostrando que a rocha pode estar sofrendo alterações em diferentes níveis. Isto porque o tipo de rocha encontrada nas fachadas do Museu, quando sã (inalterada) e não polida, apresenta valores na faixa de $780 \mathrm{HDL}( \pm 30)$ para leptinitos $(5 \times 5 \times 5 \mathrm{~cm})$.

Tabela 1. Dados referentes às médias de dureza das janelas em $H D L$

\begin{tabular}{llllllll}
\hline Corpo & C1A & C1B & C1C & C2A & C2B & C6A & C6B \\
\hline SJ1 & 386 & 440 & 522 & - & - & 486 & 508 \\
SJ2 & 328 & 434 & 414 & 358 & 471 & 450 & 434 \\
SJ3 & 344 & 396 & 380 & 370 & 408 & 461 & 502 \\
SJ4 & 398 & 382 & 322 & 245 & 291 & - & - \\
\hline
\end{tabular}

Tabela 2. Dados referentes às médias de dureza das janelas em $H D L$

\begin{tabular}{ccccccc}
\hline Corpo & C1A & C2A & C2B & C6A & C6B & C7A \\
\hline SP1 & 591 & 542 & 489 & 632 & 505 & 501 \\
SP2 & 401 & 400 & 528 & 550 & 499 & 483 \\
SP3 & 469 & 514 & 520 & 494 & 518 & 430 \\
SP4 & 421 & 512 & 587 & 422 & 547 & 427 \\
SP5 & 610 & 580 & 612 & 606 & 576 & 606 \\
\hline
\end{tabular}

\subsection{Análise química (fluorescência de raios-X)}

A Tabela 3 apresenta os valores da composição química dos corpos que constituem a janela SJ3. Os valores de alumínio e silício encontrados corroboram com a hipótese das rochas ornamentais serem leptinitos, gnaisses de coloração clara (félsicos), compostos essencialmente por quartzo (dióxido de silício- SiO2) e feldspato (silicato de alumínio, rico em potássio, sódio ou cálcio $(\mathrm{K}, \mathrm{Na}, \mathrm{Ca})(\mathrm{Si}, \mathrm{Al}) 4$, apresentando ainda um pequeno teor de granada almandina (silicato de ferro e alumínio - Fe3Al2(SiO4)3. Como o FRX faz uma análise pontual, a maioria dos resultados foi correspondente à sílica e ao quartzo, mais abundantes na rocha. Já o teor de ferro encontrado sugere a evidência de granada almandina.

Tabela 3. Composição química (\%) pela fluorescência de raios-X portátil na Janela SJ3

\begin{tabular}{cccccccc}
\hline Elemento & C1A & C1B & C1C & C2A & C2B & C6A & C6B \\
\hline Al & 31,30 & 20,30 & 18,80 & 12,80 & 16,60 & 20,50 & 16,90 \\
$\mathrm{Si}$ & 59,80 & 75,30 & 79,40 & 81,40 & 75,90 & 78,10 & 49,70 \\
$\mathrm{Ti}$ & 2,42 & 0,87 & 0,58 & 0,96 & 1,38 & 0,38 & 2,44 \\
$\mathrm{Fe}$ & 5,80 & 2,93 & 0,98 & 4,09 & 5,36 & 0,72 & 28,60 \\
Outros & 0,68 & 0,60 & 0,24 & 0,75 & 0,76 & 0,30 & 2,36 \\
\hline
\end{tabular}

\subsection{Análise colorimétrica e de brilho}

Pela análise colorimétrica e de brilho (Tabela 4) é possível observar que há uma leve alteração cromática nos corpos avaliados, corroborando com os resultados da identificação de morfologias de alteração.

Tabela 4. Distribuição colorimétrica e de brilho da janela SJ3

\begin{tabular}{cccccccccc} 
Corpo & C1A & C1B & C1C & C2A & C2B & C2C & C6A & C6B & C6C \\
\hline L* & 61,33 & 56,79 & 62,72 & 61,26 & 56,17 & 56,42 & 59,99 & 60,77 & 68,31 \\
\hline$a^{*}$ & 1,29 & 1,82 & 1,43 & 2,67 & 2,59 & 4,25 & 1,15 & 1,35 & 1,47 \\
\hline b$^{*}$ & 9,39 & 12,01 & 11,14 & 13,76 & 12,66 & 15,07 & 15,37 & 16,39 & 12,49 \\
\hline G & 0,50 & 0,57 & 0,8 & 0,63 & 0,47 & 0,50 & 0,70 & 0,70 & 1,00 \\
\hline Cor & & & & & & & & & \\
\hline
\end{tabular}

L: eixo claro/escuro, a: eixo verde/vermelho, b: eixo azul/amarelo e G: brilho

\subsection{Análise de sais e sujidades}

Na Tabela 5 estão apresentados os resultados da análise química da água de lavagem. A janela SJ4 apresentou um maior teor de íons $\mathrm{Na}+2$ e $\mathrm{Cl}$ - e podem estar relacionados com a proximidade do Museu à Baía de Guanabara (cerca de $800 \mathrm{~m}$ de distância). Já o enxofre (S2-) encontrado pode estar relacionado à poluição oriunda do intenso tráfego de veículos na rua em que se encontra a casa. Os resultados da janela SJ4, em geral, apresentaram maiores valores para quase todos os íons analisados. 
Tabela 5. Análise química (mg.L-1) dos íons encontrados na água de lavagem

\begin{tabular}{ccccccccccc}
\hline Amostra & $\mathbf{N a}^{+}$ & $\mathbf{A l}^{3+}$ & $\mathrm{Ca}^{2+}$ & $\mathbf{C l}$ & $\mathbf{K}^{+}$ & $\mathrm{Fe}^{+2}$ & $\mathbf{M g}^{+2}$ & $\mathbf{s}^{2-}$ & $\left(\mathbf{S O}_{4}\right)^{2-}$ & $\left(\mathrm{NO}_{3}\right)^{-}$ \\
\hline SJ1 & 3,2 & 0,18 & 23,2 & 7,9 & 1,8 & 0,070 & 1,20 & 17,4 & 37,0 & $<0,06$ \\
SJ2 & 4,9 & $<0,02$ & 39,4 & 5,1 & 1,6 & 0,010 & 0,69 & 5,0 & 10,0 & $<0,06$ \\
SP2 & 2,0 & $<0,02$ & 23,3 & 5,8 & 1,6 & 0,010 & 0,69 & 5,0 & 10,0 & $<0,06$ \\
SP3 & 4,8 & $<0,02$ & 30,4 & 7,0 & 1,9 & 0,005 & 1,10 & 10,5 & 20,0 & $<0,06$ \\
SP4 & 3,1 & $<0,02$ & 17,0 & 6,2 & 2,4 & 0,005 & 0,62 & 5,1 & 15,0 & 4,90 \\
SJ3 & 5,1 & $<0,02$ & 32,4 & 6,3 & 1,5 & 0,010 & 0,62 & 6,5 & 16,0 & $<0,06$ \\
SP5 & 1,8 & $<0,02$ & 16,9 & 2,8 & 1,1 & 0,020 & 0,23 & 2,4 & 6,7 & $<0,06$ \\
SJ4 & 8,9 & $<0,2$ & 83,6 & 9,4 & 2,9 & $<0,001$ & 0,96 & 70,6 & 141,0 & 1,70 \\
\hline
\end{tabular}

\subsection{Análise de pH das águas de lavagem}

Analisando os resultados de $\mathrm{pH}$, conforme Tabela 6, verificou-se a tendência de $\mathrm{pH}$ na região de basicidade, possivelmente associado à degradação das rochas pelo enxofre e formação de gipso, além da liberação de cálcio, contribuindo para o aumento dos valores de $\mathrm{pH}$.

Tabela 6. Resultados do pH de água de lavagem

\begin{tabular}{lcccccccc}
\hline Amostra & SJ1 & SJ2 & SP2 & SP3 & SP4 & SJ3 & SP5 & SJ4 \\
\hline $\mathrm{pH}$ & 7,48 & 8,25 & 6,96 & 8,04 & 7,70 & 8,29 & 7,41 & 8,10 \\
\hline
\end{tabular}

\section{CONCLUSÕES}

A maioria das rochas ornamentais da fachada principal sul do Museu Casa de Rui Barbosa, que constituem as suas portas e janelas, tratam-se possivelmente de leptinitos, cuja análise de FRX portátil evidencia a afirmação devido ao alto teor de silício ( $>50 \%$ ) e alumínio ( $>20 \%$ ), além de ferro; juntamente com o mapeamento litológico que também corrobora o parecer. As rochas apresentam modificação cromática, perda de massa, fissuras e esfoliação. Os resultados apontam que há um processo de degradação contínuo destas rochas, sendo influenciado por fatores climáticos, principalmente relacionados à proximidade com o mar e a alta concentração de enxofre depositada na fachada.

\section{AGRADECIMENTOS}

Ao CNPq e CIEE pelo apoio financeiro e ao CETEM pela infraestrutura. A todos os funcionários da Retrofit e à Fundação Casa Rui Barbosa que colaboraram com esse trabalho.

\section{REFERÊNCIAS BIBLIOGRÁFICAS}

ASTM A956 - 12, Standard Test Method for Leeb Hardness Testing of Steel Products, 2012.

NPArq - Núcleo de Preservação Arquitetônica, Centro de Memória e Informação, Caderno de Apoio à Elaboração do Caderno de Encargos: Conservação das superfícies arquitetônicas do Museu Casa de Rui Barbosa. 2015.

Contribuição ao 1‥ Simpósio Brasileiro de Caracterização e Conservação da Pedra 14 a 16 de dezembro de 2016, Congonhas - MG

Nota:

É de responsabilidade da comissão editorial do Simpósio a revisão gramatical, ortográfica, de citações e referências bibliográficas. As normas de submissão podem se diferenciar das desta revista. 